\title{
A Comparison Between Low-Level Laser Therapy and Intra-articular Ozone Injection in Knee Osteoarthritis Treatment: A Randomized Clinical Trial
}

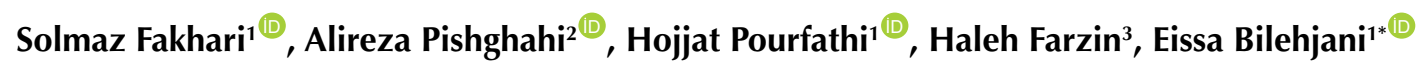 \\ 'Department of Anesthesiology, Faculty of Medicine, Tabriz University of Medical Sciences, Tabriz, Iran; \\ Anesthesiology Research Center, Tabriz University of Medical Sciences, Tabriz, Iran \\ ${ }^{2}$ Department of Physical Medicine and Rehabilitation, Faculty of Medicine, Tabriz University of Medical Sciences, \\ Tabriz, Iran \\ ${ }^{3}$ Pain and Palliative Center, Faculty of Medicine, Tabriz University of Medical Sciences, Tabriz, Iran
}

\section{*Correspondence to Eissa Bilehjani, \\ Department of anesthesiology, Faculty of Medicine, Tabriz University of Medical Sciences, Tabriz, Iran. Anesthesiology Research Center, Tabriz University of Medical Sciences, Tabriz, Iran}

Email: isadavod@gmail.com

Received: July 5, 2021

Accepted: February 2, 2021 Published online August 16, 2021

\begin{abstract}
Introduction: Knee osteoarthritis (KOA) is a common degenerative joint disease, causing deformity, pain and a limited joint range of motion. Modification of the lifestyle and an exercise training program are the cornerstone of treatment. Alternative therapies such as laser or ozone are commonly used, but there is not any comparative study of low-level laser therapy (LLLT) versus ozone therapy. The aim of the study was to compare the efficacy and safety of the LLLT versus ozone in patients with KOA.

Methods: In this single-blinded randomized clinical trial, 60 patients with KOA were assigned to LLLT or ozone groups $(n=30)$. The First basic pain severity, the Western Ontario and McMaster Universities Arthritis Index (WOMAC) score and physical function were determined. Then in the LLLT group, the patients were provided with 12 sessions of LLLT. In the ozone group, 6 sessions of intra-articular injection of ozone were organized (in each session a mixture of $10 \mathrm{~mL}$ of bupivacaine $0.25 \%$ with $15 \mathrm{~mL}$ of ozone $30 \mu \mathrm{g} / \mathrm{mL}$ ). In the middle and at the end of the intervention period, we reassessed the joint pain and physical function and the degree of improvement compared between the two groups.

Results: In the middle and at the end of the treatment period in both groups, the joint pain decreased significantly. The same as pain, the self-administrated WOMAC score and the range of joint motion improved significantly in both groups. All of these variables exposed more improvement in the ozone group patients.

Conclusion: The study showed that both LLLT and ozone are acceptable non-invasive methods in the non-surgical treatment of KOA. Compared to LLLT, the ozone was more effective. These methods must be considered in any patient who is not suitable for surgical interventions or does not experience enough improvement in symptoms following long periods of common exercise training programs.
\end{abstract}

Keywords: Knee osteoarthritis; Low-level laser therapies; Ozone therapy.

\section{Introduction}

Knee osteoarthritis (KOA) is a common degenerative joint disease, which is manifested by pain, a limited range of motion, and joint deformity that may lead to disability in walking and limited daily activity. It is the consequence of an imbalance between regenerative and degenerative activities in the joint space. ${ }^{1-6}$ Females are more vulnerable than males to this disease. ${ }^{2}$ Obesity, gender, age, genetic background, repeated trauma, and mechanical load are predisposing factors of KOA. ${ }^{7,8} \mathrm{KOA}$ is characterized by joint cartilage damage, subchondral sclerosis and cysts, joint space narrowing, marginal osteophytes, and joint deformity. ${ }^{9}$ The joint cartilage has limited regenerative and repairing capacity. This is due to a limited cell count of mineral tissue, a low rate of metabolism, and limited amounts of matrix fibers and vascular supply., ${ }^{40}$ Inflammatory cytokines (interleukins 1, 6 and 8, tumor necrosis factor $\alpha$, interferon-gamma) and oxidative damage are the main factors in cartilage damage in KOA. ${ }^{4}$ The novel options for the treatment of KOA involve targeting the inflammatory pathways in order to switch the degenerative environment of the

Please cite this article as follows: Fakhari S, Pishghahi A, Pourfathi H, Farzin H, Bilehjani E. A comparison between low-level laser therapy and intra-articular ozone injection in knee osteoarthritis treatment: a randomized clinical trial. J Lasers Med Sci. 2021;12:e44. doi:10.34172/jlms.2021.44 
knee to a regenerative one. The Western Ontario and McMaster Universities Arthritis Index (WOMAC) is a common and widely used scoring method that is used to evaluate the severity of KOA. In this method by a self-administered questionnaire, the pain, stiffness and physical function of the joints are evaluated. It measures 5 items for pain (score range: 0-20), 2 for stiffness (score range: $0-8$ ), and 17 for functional limitation (score range: $0-68$ ). These scores are summed up; a higher total WOMAC score indicates worse pain, more joint stiffness and functional limitations. Considering this scoring method, the disorder severity was classified as mild (grade I), moderate (grade II), severe (grade III), and extreme (grade IV). ${ }^{11}$ The severity of the KOA was classified radiographically in grades I-IV based on the Kellgren-Lawrence grading method. ${ }^{12}$

Various methods have been reported for the management of $\mathrm{KOA}$ in the literature. Nonsurgical treatments are the first step. Surgical options are considered for patients who continue to have knee pain 6 months after non-operative treatments. ${ }^{13,14}$ Modification of the lifestyle, weight control, and an exercise training program are the cornerstone of treatment. Oral and intraarticular medications, physiotherapy, and even alternative medicine are other treatment modalities. The surgical approach (joint replacement surgery) is used in chronic, severe, and refractory cases. ${ }^{8,15,16}$ It seems that none of these approaches are superior to each other and should be applied individually for each patient. ${ }^{17,18}$

Low-level laser therapy (LLLT) and intra-articular ozone injection both are used in pain medicine. LLLT is a method that uses a low-level laser (LLL) for the induction of cartilage repairing and pain modulation in KOA. It is believed that LLLT may increase mitochondrial ATP levels and enhance tissue oxygenation. It might also have analgesic effects through the increased level of neurotransmitters such as serotonin involved in pain modulation. ${ }^{8}$ A low-level laser has a proliferative effect on fibroblasts and osteoblasts and modulates inflammatory markers such as PGE2, mRNA Cox 2 , IL-1 $\beta$, TNFa, ${ }^{19-21}$ cytokines such as interleukin-1 alpha and interleukin-1 beta, and fibroblast growth factor. ${ }^{21-23}$ LLLT is able to increase cell numbers, DNA, and RNA synthesis and collagen production, initiate mitosis in cultured cells and convert light energy into chemical energy in the form of ATP within the cell, which enhances cellular functions and cell proliferation rates. ${ }^{24}$ Stausholm et al. in meta-analyses on KOA treatment concluded the pain relief by LLLT compared to the placebo. ${ }^{25}$ Rayegani et al investigated the effect of the LLLT on KOA and showed it reduced pain, joint stiffness and disability and it was superior to the placebo and therapeutic ultrasound. ${ }^{9}$ In a study, it was concluded that in older patients with chronic KOA, the addition of LLLT to an exercise training program was more effective than exercise training alone. ${ }^{26}$
Ozone (O3) is a gas with anti-inflammatory, antimicrobial and immunomodulatory effects. It is associated with increased tissue oxygenation and circulation by the induction of vasodilation and angiogenesis, the elimination of inflammatory metabolic products from joint space, the normalization of intracellular antioxidants and nitric-oxide levels and the reduction of superoxide production..$^{27,28}$ Ozone activates the enzymes which are responsible for protecting against the overproduction of superoxide processes. ${ }^{15}$ Ozone has a bioregulatory role through cytokines such as IL-8, TNF-a, TGF betal and tissue remodeling. ${ }^{15}$ Ozone may create the right context for adaptation to oxidative stress or promote oxidative preconditioning which stimulates the antioxidant endogenous systems resulting in the protection against tissue damage. ${ }^{29,30}$ Studies have shown the intra-articular ozone injection can relieve the symptoms of KOA. ${ }^{7,31}$ In a systematic review, Sconza et alconcluded ozone was able to provide significant pain relief in $\mathrm{KOA}$, at least at a short-term follow-up. ${ }^{32}$

Although laser and ozone therapy have been used in a lot of pain studies, only a few studies have compared these two treatment modalities with each other. Kazancioglu and Erisen, in a randomized study, reported that topical ozone is more effective than LLLT for pain relief in patients with oral lichen planus. ${ }^{33}$ In an animal study conducted on rats, LLLT had a more protective effect on bone destruction, when compared with ozone therapy. ${ }^{34}$ With the aim of pain relief after orthodontic surgery, LLLT was effective, but ozone was not. ${ }^{35}$ Considering these limited data, we decided to search any published comparative studies into ozone therapy versus LLLT. However, there was not any published study. Thus, we designed the present study and investigated the efficacy and safety of the intra-articular ozone injection versus LLLT in the management of KOA pain.

\section{Methods and Materials}

This study was registered by the Iranian randomized clinical trial organization (Identifier: IRCT20180518039700N1). The authors obtained written informed consent from all of the patients. Sixty patients, who were suffering from $\mathrm{KOA}$ and considered to get non-surgical treatment in our pain clinic, randomly entered this study in two groups: ozone group or LLLT group $(n=30)$. The sample size was calculated using power \& sample size software (ver. 3.1 ); considering $\alpha=0.05$, power of study 0.80 , in purpose of discovering $30 \%$ differences between two treatment morality due to knee pain and function scores.

Entering the study, the patient was visited by an anesthesiologist with the aim of discovering the disease severity before any intervention. She was kept blind to the type of intervention throughout the study. She searched the basic WOMAC score and joint mobility. The WOMAC score was defined using the Persian version of 
the self-administered WOMAC questionnaire and joint mobility in active flexion, measured by a goniometer. ${ }^{36}$

The patients of both genders, aged above 45 years, were eligible to participate in this investigation. They had a radiographic report of the knee OA of grade II, III based on the Kellgren-Lawrence grading method. Patients complained of pain and decreased knee function and failure to respond to conventional treatments during the past three months. In patients with bilateral $\mathrm{KOA}$, the intervention was carried out on only one joint.

Patients were excluded if they met the following criteria: severe knee OA (Kellgren-Lawrence grade 4), joint deformity or ankylosis, a history of corticosteroid consumption during the past 3 months, a history of rheumatoid diseases, coagulopathy, previous knee surgery, and acute infection. In the occurrence of any contraindication to LLLT (malignancy, epilepsy, pregnancy, heart pacemaker) or ozone therapy (hyperthyroidism, thrombocytopenia, and recent heart attack), the patients were excluded from the study.

The patients in the LLLT group were treated with 12 sessions of LLLT (3 sessions per week). The BTL4000 branded laser device was used with calibration of producing $980 \mathrm{NM}$ continuous wave pulses $(980 \mathrm{~nm})$, with an intensity of $6 \mathrm{~J} /$ point, duration of 150 seconds/ point and beam area of $0.5 \mathrm{~cm}^{3}$. During each session, 7 points around the knee joint were treated: 2 points on the medial, 2 points on the lateral borders and 3 points on the anterior aspect of the patella. In the ozone group, we administered 6 sessions of intra-articular ozone injection ( 3 sessions per week). In each session, a mixture of $10 \mathrm{ml}$ of bupivacaine $0.25 \%$ with $15 \mathrm{~mL}$ of ozone $30 \mu \mathrm{g} / \mathrm{mL}$ was injected into the knee joint through the inferior-medial approach in a supine position with a semi-flexed knee. Applying a few joint flexion/extension, we tried to better spread the solution. ${ }^{27}$ All of the laser therapies and intraarticular injections were done by an anesthesiologist skilled in pain medicine.

The primary outcomes of the study were the severity of the pain and the physical function of the knee. Thus, in the middle and at the end of the intervention period, the anesthesiologist visited the patients again and re-assessed their clinical and functional status using the visual analog scale (VAS) pain scale method, WOMAC questionnaire and knee joint goniometry. The anesthesiologist was blind to the patient's intervention group.

\section{Statistical Analysis}

The collected data were analyzed using SPSS statistical software (version 22.0 SPSS Inc., Chicago, IL, USA). The Kolmogorov-Smirnov test was applied to check the distribution normality of quantitative parameters. Quantitative variables were presented as mean \pm SD and categorical variables were presented as frequencies (percentage). We used student's t-test for independent groups and repeated measures ANOVA test to compare the normally distributed data between or within groups respectively. The $\chi^{2}$ or Fisher's exact tests were used to compare the categorical data between two groups and the Mann-Whitney test was used to compare the categorical data within groups. These tests were performed as twosided test and $P$ values $<0.05$ were considered statistically significant.

\section{Results}

No patients were missed for follow-up, and the data of 60 patients were analyzed and compared between the two groups. Most of the patients were female (38 females vs. 22 male). Demographic characteristics are presented in Table 1. They were similar for both groups (Table 1). The right side KOA was more common than the left side ( $55 \%$ vs. $45 \%$ respectively), and it was distributed equally between the two groups $(P$ value $=0.79)$.

The basic pain intensity that was evaluated by the VAS was the same before any intervention ( $P$ value 0.087 ) Compared to the basic pain values, after initiating the intervention, there was a significant decrease in pain intensity in both ozone and LLLT groups (both in the middle and at the end of the treatment phases, $P$ value $=0.001)$ (Table 2). Comparing the two groups with each other revealed that the intervention in the ozone group had a more therapeutic effect on pain relief (both in the middle and at the end of the treatment phases, $P$ value $=0.001$ ).

The basic WOMAC score was similar in the ozone and LLLT groups. $50.03 \pm 2.87$ and $49.5 \pm 2.02$ respectively

Table 1. The Demographic Characteristics of the Participants

\begin{tabular}{|c|c|c|c|c|c|}
\hline & & Ozone group $(n=30)$ & LLLT group $(n=30)$ & Total $(n=60)$ & $P$ value \\
\hline \multirow{2}{*}{ Gender } & Male n (\%) & $10(33.3 \%)$ & $12(40 \%)$ & $22(36.7)$ & \multirow{2}{*}{0.592} \\
\hline & Female $\mathrm{n}(\%)$ & $20(66.6 \%)$ & $18(60 \%)$ & $38(63.3 \%)$ & \\
\hline Age mean (SD) & & $57.67 \pm 9.35$ & $53.33 \pm 9.37$ & $55.5 \pm 9.36$ & 0.078 \\
\hline Height mean (SD) & & $166.9 \pm 6.9$ & $166.2 \pm 4.4$ & $166.55 \pm 5.25$ & 0.841 \\
\hline Weight mean (SD) & & $87.53 \pm 12.4$ & $92.0 \pm 6.1$ & $89.77 \pm 9.87$ & 0.083 \\
\hline \multirow{2}{*}{$\begin{array}{l}\text { Side of treated knee } \\
\text { joint }\end{array}$} & Right & $17(56.7 \%)$ & $16(53.3 \%)$ & $33(55.0 \%)$ & \multirow{2}{*}{0.79} \\
\hline & Left & $13(43.3 \%)$ & $14(46.7 \%)$ & $27(45.0 \%)$ & \\
\hline
\end{tabular}


$(P$ value $=0.088)$. Compared to the WOMAC score, after initiating the intervention, there was a significant improvement in the joint function in both ozone and LLLT groups (both in the middle and at the end of the treatment phases, $P$ value $=0.048$ ). Comparing two groups with each other, the ozone therapy caused a definite improvement in joint function (both in the middle and at the end of the treatment phases, $P$ value $=0.004)($ Table 3$)$.

Table 4 shows the improvement in the measured arc of joint flexion. Both the ozone or laser therapy exposed a significant positive effect on the range of joint motion. Any time in the study, this effect was more dominant in the ozone group $(P$ value $<0.05)$.

\section{Discussion}

The present study compared the two important alternative therapies of $\mathrm{KOA}$ in order to induce improvement in physical function and pain relief. It showed that both the intra-articular ozone and laser therapy were effective treatment modalities; however, the intra-articular injection of ozone was a more effective method. In the previous studies, the effects of laser or ozone therapy were investigated in order to manage $\mathrm{KOA}$, but there is not any study in which these two methods have been compared with each other in KOA treatment. In a study conducted by Youssef et al, LLLT was added to a common exercise training program of chronic KOA treatment. They concluded that the addition of LLLT (especially $6 \mathrm{~J} / \mathrm{cm}^{2}$ ) to an exercise training program could be more effective than exercise training alone. ${ }^{26}$

Rayegani et al compared the therapeutic effect of the LLLT or ultrasonic therapy with the placebo in patients suffering from KOA. They concluded that both of these alternative therapies were able to reduce symptoms and improve physical function. In this way, LLLT was superior to ultrasonic therapy. ${ }^{9}$

Ozone $\left(\mathrm{O}_{3}\right)$ is a type of oxygen molecule. Compared to the usual molecule of oxygen $\left(\mathrm{O}_{2}\right)$, it is very unstable due to the strong tendency to react with other molecules. It may act as hyperbaric oxygen in the body with a capability of spontaneous conversion to $\mathrm{O}_{2}$ with releasing an oxygen free radical. In contrast to the dogma that this free radical is always toxic to the body, decades of clinical experience have shown that the oxygen free radical can act as a disinfectant, a metabolic enhancer, a paradoxical inducer of antioxidant enzymes, an immunomodulator, an inducer of endothelial nitric oxide synthase and possibly an activator of stem cells with consequent

Table 2. Knee Pain Score by Visual Analog Scale

\begin{tabular}{lccccc}
\hline & Time point & Ozone group $(\mathbf{n}=\mathbf{3 0})$ & LLLT group $(\mathbf{n}=\mathbf{3 0})$ & Total $(\mathbf{n}=\mathbf{6 0})$ & $\boldsymbol{P}$ Value** \\
\hline \multirow{3}{*}{ Pain score } & basic & $9.23 \pm 0.89$ & $9.60 \pm 0.81$ & $9.42 \pm 0.84$ & 0.087 \\
& Mid of treatment & $6.01 \pm 1.43$ & $8.00 \pm 0.64$ & $7.05 \pm 1.08$ & 0.0001 \\
& End of study & $3.67 \pm 1.24$ & $6.60 \pm 1.04$ & $5.14 \pm 1.16$ & 0.001 \\
\hline value* & & 0.001 & 0.001 & 0.001 & - \\
\hline
\end{tabular}

* Within group $P$ values.

** Between group $P$ values.

Table 3. The WOMAC Score Changes in the Ozone Group Versus the LLLT Group

\begin{tabular}{|c|c|c|c|c|c|}
\hline & Time Point & Ozone Group $(n=30)$ & LLLT Group $(n=30)$ & Total $(n=60)$ & $P$ Value** \\
\hline \multirow{3}{*}{ WOMAC score } & basic & $50.03 \pm 2.87$ & $49.5 \pm 2.02$ & $49.91 \pm 2.32$ & 0.088 \\
\hline & Mid of treatment & $37.01 \pm 2.22$ & $42.31 \pm 1.32$ & $39.66 \pm 1.75$ & 0.045 \\
\hline & End of study & $27.61 \pm 2.01$ & $35.24 \pm 1.53$ & $31.45 \pm 1.74$ & 0.004 \\
\hline$P$ value* & & 0.001 & 0.001 & 0.001 & \\
\hline
\end{tabular}

* Within group $P$ values.

** Between group $P$ values.

Table 4. Changes in the Measured Range of Knee Joint Flexion in the Ozone Group Versus the LLLT Group

\begin{tabular}{|c|c|c|c|c|c|}
\hline & Time Point & Ozone Group $(n=30)$ & LLLT Group $(n=30)$ & Total $(n=60)$ & $P$ Value* \\
\hline \multirow{3}{*}{$\begin{array}{l}\text { Goniometric } \\
\text { range }\end{array}$} & basic & $120.23 \pm 4.37$ & $118.33 \pm 5.36$ & $119.16 \pm 5.41$ & 0.137 \\
\hline & Mid of treatment & $124.03 \pm 4.83$ & $121.12 \pm 5.41$ & $122.56 \pm 5.12$ & 0.031 \\
\hline & End of study & $129.90 \pm 5.71$ & $124.37 \pm 6.79$ & $127.32 \pm 6.15$ & 0.001 \\
\hline$P$ value** & & 0.002 & 0.049 & 0.015 & \\
\hline
\end{tabular}

* Within group $P$ values.

** Between group $P$ values. 
neovascularization and tissue reconstruction. ${ }^{37}$ Indeed, ozone may increase localized blood circulation and oxygen load in traumatized or damaged tissues such as joint cartilage, ligament, or tendon and eventually reestablish the healing process. Ozone also has some effects on growth factors. It can stimulate their synthesis, especially endothelial growth factors; thus enhance their receptors on cell membranes and facilitate their actions. ${ }^{38}$ In a randomized clinical trial, comparing intra-articular injection of ozone to methylprednisolone in patients with $\mathrm{KOA}$, the pain relief was more meaningful in the ozone group. ${ }^{7}$ Nowadays, ozone injection is not limited only to joint disorders, but there are reports of its beneficial effects on soft tissue (tendon, ligament, and muscles) diseases as well as inter-vertebral disc disorders. ${ }^{39}$ On the other hand, there are some conflicting findings due to the efficacy of the LLLT in KOA. ${ }^{25,40}$ This may be due to the differences in the administration protocols of the LLLT. There is no consensus regarding the pulse duration, pulse width, wavelength, pulse-duty time, treatment duration in each session, treatment frequency, and probe location in LLLT. Various factors such as the severity of OA, osteophyte formation, joint cartilage thickness, and even the joint range of motion may be important in the response to laser therapy. Our study supported the effectiveness of the LLLT; however, compared with intra-articular ozone, it was a less effective treatment. Therefore, the authors believe that both LLLT and intra-articular ozone must be considered acceptable, safe and non-invasive methods of KOA non-surgical treatment.

\section{Conclusion}

Our study showed that both LLLT and intra-articular ozone are acceptable, safe and non-invasive methods in the non-surgical treatment of KOA. However, compared to intra-articular ozone, LLLT was a less effective treatment modality. These methods must be considered in any patient who is not suitable for invasive surgical interventions or does not get enough improvement in his or her symptoms following long periods of common exercise training programs.

\section{Limitations}

The present study had some limitations such as a small sample size and lack of a placebo group (we considered it not ethical to have a placebo group). The short period of the study and lack of a long follow-up period were other limitations, and thus we cannot get any information due to the persistence of therapeutic effects of any intervention. We added bupivacaine to ozone; however, it has a lifetime of 12-24 hours, and it may work as a confounding variable.

\section{Ethical Considerations}

This study was performed after obtaining approval of the local institutional ethical committee (Tabriz University of Medical Sciences; registry number: TBZMED.REC.1396.1097)

\section{Conflict of Interests}

The authors declare that there are not any conflicts of interest in this work.

\section{Funding}

The authors stated that they had no funding support.

\section{References}

1. Calunga JL, Menéndez S, León R, Chang S, Guanche D, Balbín A, et al. Application of ozone therapy in patients with knee osteoarthritis. Ozone Sci Eng. 2012;34(6):469-75. doi: 10.1080/01919512.2012.719120

2. de Meneses SRF, Hunter DJ, Docko EY, Marques AP. Effect of low-level laser therapy (904 nm) and static stretching in patients with knee osteoarthritis: a protocol of randomised controlled trial. BMC Musculoskelet Disord. 2015;16(1):1-9. doi: 10.1186/s12891-015-0709-9

3. Kanzaki N, Ono Y, Shibata H, Moritani T. Glucosaminecontaining supplement improves locomotor functions in subjects with knee pain: a randomized, double-blind, placebo-controlled study. Clin Interv Aging. 2015;10:1743. doi: 10.2147/CIA.S93077

4. Fernandez-Cuadros ME, Perez-Moro O, Mirón-Canelo JA. Could ozone be used as a feasible future treatment in osteoarthritis of the knee. Divers Equal Health Care. 2016;13(3):232-9. doi: 10.21767/2049-5471.100057

5. Kobsar D, Osis ST, Hettinga BA, Ferber R. Gait biomechanics and patient-reported function as predictors of response to a hip strengthening exercise intervention in patients with knee osteoarthritis. PLoS One. 2015;10(10):e0139923. doi: 10.1371/journal.pone.0139923

6. Hashemi M, Jalili P, Mennati S, Koosha A, Rohanifar R, Madadi F, et al. The effects of prolotherapy with hypertonic dextrose versus prolozone (intraarticular ozone) in patients with knee osteoarthritis. Anesth Pain Med. 2015;5(5). doi: 10.5812/aapm.27585

7. Mishra SK, Pramanik R, Das P, Das PP, Palit AK, Roy J, et al. Role of intra-articular ozone in osteo-arthritis of knee for functional and symptomatic improvement. J Pain Res. 2011;22(2):65-9. doi: 10.2147/JPR.S142755

8. Fukuda VO, Fukuda TY, Guimarães M, Shiwa S, de Lima BDC, Martins RÁBL, et al. Short-term efficacy of lowlevel laser therapy in patients with knee osteoarthritis: a randomized placebo-controlled, double-blind clinical trial. Rev Bras Ortop. 2011;46(5):526-33. doi: 10.1016/S22554971(15)30407-9

9. Rayegani SM, Bahrami MH, Elyaspour D, Saeedi M, Sanjari H. Therapeutic effects of low level laser therapy (LLLT) in knee osteoarthritis, compared to therapeutic ultrasound. J Lasers Med Sci. 2012. doi:10.22037/JLMS.V3I2.2830

10. Manoto S, Maepa M, Motaung S. Medical ozone therapy as a potential treatment modality for regeneration of damaged articular cartilage in osteoarthritis. Saudi J Biol Sci 2018; 25 (4): 672-9. doi: 10.1016/j.sjbs.2016.02.002

11. Lequesne $M$, Mery C, Samson M, Gerard P. Indexes of severity for osteoarthritis of the hip and knee: validation-value in comparison with other assessment tests. Scand J Rheumatol Suppl. 1987;16(sup65):85-9. doi: 10.3109/03009748709102182

12. Kohn MD, Sassoon AA, Fernando ND. Classifications in 
brief: Kellgren-Lawrence classification of osteoarthritis. Clin Orthop Relat Res. Springer; 2016. doi: 10.1007/s11999016-4732-4

13. The Editors of Encyclopedia Britannica. Leaf-nosed bat. In: Encyclopedia Britannica [Internet]. 2009. Available from: https://www.britannica.com/animal/leaf-nosed-bat

14. Zhang W, Moskowitz R, Nuki G, Abramson S, Altman $\mathrm{RD}$, Arden N, et al. OARSI recommendations for the management of hip and knee osteoarthritis, Part II: OARSI evidence-based, expert consensus guidelines. Osteoarthr Cartil. 2008;16(2):137-62. doi: 10.1016/j.joca.2007.12.013

15. Manoto SL, Maepa MJ, Motaung SK. Medical ozone therapy as a potential treatment modality for regeneration of damaged articular cartilage in osteoarthritis. Saudi J Biol Sci. 2018;25(4):672-9. doi: 10.1016/j. sjbs.2016.02.002

16. Shengelia R, Parker SJ, Ballin M, George T, Reid MC. Complementary therapies for osteoarthritis: are they effective? Pain Manag Nurs. 2013;14(4):e274-e88. doi: 10.1016/j.pmn.2012.01.001

17. Buckwalter JA, Stanish WD, Rosier RN, Schenck Jr RC, Dennis DA, Coutts RD. The increasing need for nonoperative treatment of patients with osteoarthritis. Clin Orthop Relat Res. 2001; 385:36-45. doi: 10.1097/00003086-200104000-00008

18. Samson DJ, Grant MD, Ratko TA, Bonnell CJ, Ziegler KM, Aronson N. Treatment of primary and secondary osteoarthritis of the knee. Evid Rep Technol Assess (Full Rep). 2007(157):1-157.

19. Bjordal JM, Johnson MI, Iversen V, Aimbire F, LopesMartins RAB. Low-level laser therapy in acute pain: a systematic review of possible mechanisms of action and clinical effects in randomized placebo-controlled trials. Photomed Laser Surg. 2006;24(2):158-68. doi: 10.1089/ pho.2006.24.158

20. Tamura K, Hosoya S, Hiratsuka K, Abiko Y. Laser stimulation of CDC46 gene expression in murine osteoblasts. Photomed Laser Surg. 1998; 10:25-31. doi: 10.1089/pho.2008.2297

21. Hegedűs B, Viharos L, Gervain M, Gálfi M. The effect of low-level laser in knee osteoarthritis: a double-blind, randomized, placebo-controlled trial. Photomed Laser Surg. 2009;27(4):577-84. doi: 10.1089/pho.2008.2297

22. Bettencourt F. Effects of Class IV Laser in Knee Osteoarthritis: A Randomized Control Trial. J Arthr. 2020;9(1):1-5.

23. Montes-Molina R, Madronero-Agreda MA, RomojaroRodriguez AB, Gallego-Mendez V, Prados-Cabiedas C, Marques-Lucas C, et al. Efficacy of interferential low-level laser therapy using two independent sources in the treatment of knee pain. Photomed Laser Surg. 2009;27(3):467-71. doi: 10.1089/pho.2008.2315

24. AlGhamdi KM, Kumar A, Moussa NA. Low-level laser therapy: a useful technique for enhancing the proliferation of various cultured cells. Lasers Med Sci. 2012;27(1):23749. doi: 10.1007/s10103-011-0885-2

25. Stausholm MB, Naterstad IF, Joensen J, Lopes-Martins RÁB, Sæbø H, Lund H, et al. Efficacy of low-level laser therapy on pain and disability in knee osteoarthritis: systematic review and meta-analysis of randomised placebo-controlled trials. BMJ open. 2019 Oct 28;9(10):e031142. doi:10.1136/ bmjopen-2019-031142

26. Youssef EF, Muaidi QI, Shanb AA. Effect of laser therapy on chronic osteoarthritis of the knee in older subjects. J Lasers Med Sci. . 2016;7(2):112-9. doi: 10.15171/jlms.2016.19

27. Camelia C, Madalina I, Tatiana M, Marilena P, Oana A. The role of ozone therapy in maintaining the articular function and in relieving the pain for patients with knee osteoarthritis. ARS Medica Tomitana. 2014;20(1):25-9. doi:10.2478/arsm-2014-0005

28. Elvis A, Ekta J. Ozone therapy: A clinical review. J Nat Sci Biol Med. 2011;2(1):66. doi: 10.4103/0976-9668.82319

29. Ajamieh $\mathrm{H}$, Menéndez $\mathrm{S}$, Martínez-Sánchez G, Candelario-Jalil E, Re L, Giuliani A, et al. Effects of ozone oxidative preconditioning on nitric oxide generation and cellular redox balance in a rat model of hepatic ischaemia-reperfusion. Liver Int. 2004;24(1):55-62. doi: 10.1111/j.1478-3231.2004.00885.x

30. Guven A, Gundogdu G, Sadir S, Topal T, Erdogan E, Korkmaz A, et al. The efficacy of ozone therapy in experimental caustic esophageal burn. J Pediatr Surg. 2008;43(9):1679-84. doi: 10.1016/j.jpedsurg.2008.01.064

31. Lopes de Jesus CC, dos Santos FC, de Jesus LMOB, Monteiro I, Sant'Ana MSSC, Trevisani VFM. Comparison between intra-articular ozone and placebo in the treatment of knee osteoarthritis: A randomized, double-blinded, placebo-controlled study. PLoS One. 2017;12(7):e0179185. doi: 10.1371/journal.pone.0179185

32. Sconza C, Respizzi S, Virelli L, Vandenbulcke F, Iacono F, Kon E, et al. Oxygen-ozone therapy for the treatment of knee osteoarthritis: a systematic review of randomized controlled trials. Arthroscopy. 2020;36(1):277-86. doi: 10.1016/j.arthro.2019.05.043

33. Kazancioglu HO, Erisen M. Comparison of low-level laser therapy versus ozone therapy in the treatment of oral lichen planus. Ann Dermatol. 2015;27(5):485-91. doi: 10.5021/ ad.2015.27.5.485

34. Bayer Alinca S, Sağlam E, Zengin Celik T, Hacisalihoglu P, Doğan M. Is low level laser therapy or ozone therapy more effective for bone healing? Understanding the mechanisms of HIF-1a, RANKL and OPG. Biotech Histochem. 2020:1-8. doi: 10.1080/10520295.2020.1743360

35. Matys J, Jaszczak E, Flieger R, Kostrzewska-Kaminiarz K, Grzech-Leśniak K, Dominiak M. Effect of ozone and diode laser $(635 \mathrm{~nm})$ in reducing orthodontic pain in the maxillary arch-a randomized clinical controlled trial. Lasers Med Sci. 2020;35(2):487-96. doi: 10.1007/s10103019-02896-0

36. Eftekhar-Sadat B, Niknejad-Hosseyni SH, Babaei-Ghazani A, Toopchizadeh V, Sadeghi H. Reliability and validity of Persian version of Western Ontario and McMaster Universities Osteoarthritis index in knee osteoarthritis. J Anal Res Clin Med 2015;3(3):170-7. doi: 10.15171/ jarcm.2015.027

37. Bocci V. The Clinical Application of Ozonetherapy. Ozone: Springer. 2010. p. 97-232. doi: 10.1007/978-90-481-92342_9

38. Shallenberger F. Prolozone ${ }^{\mathrm{TM}}$ - Regenerating Joints and Eliminating Pain. J Proloth. 2011;3(2):630-8. Published March 21, 2012. https://journalofprolotherapy.com/ 
prolozone-regenerating-joints-and-eliminating-pain/. Accessed July 5, 2021.

39. Andreula CF, Simonetti L, De Santis F, Agati R, Ricci R, Leonardi M. Minimally invasive oxygen-ozone therapy for lumbar disk herniation. AJNR Am J Neuroradiol. 2003;24(5):996-1000.
40. Rayegani SM, Raeissadat SA, Heidari S, Moradi-Joo M. Safety and effectiveness of low-level laser therapy in patients with knee osteoarthritis: a systematic review and meta-analysis. J Lasers Med Sci. 2017;8(Suppl 1): S12-19. doi: 10.15171/jlms.2017.s3 